\title{
Costimulation controls diabetes by altering the balance of pathogenic and regulatory $T$ cells
}

\author{
Hélène Bour-Jordan, ${ }^{1}$ Benoît L. Salomon, ${ }^{2}$ Heather L. Thompson, ${ }^{1}$ Gregory L. Szot, ${ }^{1}$ \\ Matthew R. Bernhard, ${ }^{1}$ and Jeffrey A. Bluestone ${ }^{1}$ \\ 1UCSF Diabetes Center, University of California at San Francisco, San Francisco, California, USA. ${ }^{2}$ Ben May Institute for Cancer Research, \\ University of Chicago, Chicago, Illinois, USA.
}

\begin{abstract}
The development of autoimmune diabetes in the nonobese diabetic (NOD) mouse results from a breakdown in tolerance to pancreatic islet antigens. CD28-B7 and CD40 ligand-CD40 (CD40L-CD40) costimulatory pathways affect the development of disease and are promising therapeutic targets. Indeed, it was shown previously that diabetes fails to develop in NOD-B7-2-/- and NOD-CD40L $\mathrm{L}^{-/-}$mice. In this study, we examined the relative role of these 2 costimulatory pathways in the balance of autoimmunity versus regulation in NOD mice. We demonstrate that initiation but not effector function of autoreactive $\mathrm{T}$ cells was defective in NOD-B7-2 ${ }^{-/-}$mice. Moreover, the residual proliferation of the autoreactive cells was effectively controlled by CD28-dependent $\mathrm{CD}^{+} \mathrm{CD}^{+} 5^{+}$regulatory $\mathrm{T}$ cells (Treg's), as depletion of Treg's partially restored proliferation of autoreactive $T$ cells and resulted in diabetes in an adoptive-transfer model. Similarly, disruption of the CD28-B7 pathway and subsequent Treg deletion restored autoimmunity in NOD-CD40 $\mathrm{L}^{-/-}$mice. These results demonstrate that development of diabetes is dependent on a balance of pathogenic and regulatory $T$ cells that is controlled by costimulatory signals. Thus, elimination of Treg's results in diabetes even in the absence of costimulation, which suggests a need for alternative strategies for immunotherapeutic approaches.
\end{abstract}

\section{Introduction}

The nonobese diabetic (NOD) mouse is the best-characterized model for type I diabetes in humans (1). Diabetes in NOD mice is mediated by inflammatory autoreactive $\mathrm{T}$ cells that recognize pancreatic islet antigens and escape central and peripheral tolerance. Pancreatic islet antigens are initially presented to pathogenic $\mathrm{T}$ cells in the pancreatic lymph nodes (LNs) (2), leading to their activation, expansion, and infiltration into the pancreas, where they induce extensive islet damage. Dampening autoreactive T cells has thus become a primary therapeutic goal in diabetes as well as other $\mathrm{T}$ cell-mediated autoimmune diseases and transplantation (3). The seminal discovery that costimulation was essential for mounting efficient $\mathrm{T}$ cell responses generated great interest and has led to therapeutic approaches aimed at costimulatory pathways (4).

Two major costimulatory pathways have been described for pathogenic T cell activation in NOD mice: the CD28-B7 and the CD40 ligand-CD40 (CD40L-CD40) pathways. CD28 interactions with the structurally related ligands B7-1 and B7-2 differentially regulate immune responses (4). In fact, treatment of NOD mice with anti-B7-2 mAb's decreased incidence of diabetes (5), and NOD mice deficient for B7-2 (NOD-B7-2-/-) were completely protected from diabetes (6). Similarly, treatment of NOD mice with anti-CD40L mAb's (7) or genetic disruption of CD40L (NOD$\left.\mathrm{CD}_{40 \mathrm{~L}^{-/-}}\right)(8,9)$ prevented the early stages of $\mathrm{T}$ cell activation in the pancreatic LNs and abrogated disease (7-9). In addition, previ-

Nonstandard abbreviations used: APC, antigen-presenting cell; CD40L, CD40 ligand; CFSE, 5-(and-6)-carboxyfluorescein diacetate, succinimidyl ester; IGRP, isletspecific glucose-6-phosphatase catalytic subunit-related protein; LN, lymph node; NOD, nonobese diabetic; TCR, T cell receptor; Tg, transgenic; Treg, regulatory T cell. Conflict of interest: The authors have declared that no conflict of interest exists.

Citation for this article: J. Clin. Invest. 114:979-987 (2004)

doi:10.1172/JCI200420483 ous studies have shown that the CD28 and CD40L pathways are linked, since CD40 engagement promotes the upregulation of B7 on antigen-presenting cells (APCs), and overexpression of B7 on APCs can bypass the need for CD40 engagement $(10,11)$. These observations suggested that the two costimulatory pathways control autoimmunity via a common pathway and that disruption of one or both would be expected to lead to similar outcomes.

$\mathrm{CD} 4{ }^{+} \mathrm{CD} 25^{+}$regulatory $\mathrm{T}$ cells (Treg's) play a fundamental role in controlling autoimmunity in the periphery $(12,13)$. We and others have shown that diabetes in the NOD mouse results from a breakdown in the balance between autoreactivity and immune regulation in the periphery (14-16). Although NOD mice are characterized by low numbers of Treg's (15), these Treg's provide a certain level of protection to NOD mice (14). In addition, it has become increasingly clear that CD28 engagement is essential for maintaining a functional Treg compartment $(14,17)$. Indeed, whereas blockade of CD28-B7 interactions in NOD mice by antagonist therapy (CTLA4Ig or anti-B7-1 plus anti-B7-2 mAb's) or gene targeting (CD28-/- and B7-1/B7-2/- mice) decreases activation of pathogenic $T$ cells on a per-cell basis, the disruption of the CD28-B7 pathway causes a much more profound defect in Treg's and results in exacerbated diabetes $(14,18)$. As a consequence, proposed therapeutic approaches for type I diabetes that efficiently target the CD28-B7 pathway have raised concerns.

In this study, we further analyzed the relative role of CD28-B7 and CD40L-CD40 in autoreactivity versus immune regulation. The absence of diabetes in NOD-B7-2-/- and NOD-CD $40 \mathrm{~L}^{-/}$ mice provided a unique opportunity to test the hypothesis that selective blockade of CD28 versus CD40L costimulatory pathways would differentially alter immune responses in NOD mice. Our results show that a diabetogenic $\mathrm{T}$ cell repertoire is present in NOD-B7-2-/- mice but that activation of islet antigen-specific 
$\mathrm{T}$ cells in the pancreatic LNs is altered due to B7-2 deficiency. In addition, the residual autoreactive $\mathrm{T}$ cells are prevented from promoting diabetes by functionally competent CD28-dependent $\mathrm{CD} 4^{+} \mathrm{CD} 25^{+}$Treg's. Finally, we demonstrated that CD28 deficiency dramatically decreased the number of Treg's and partially restored diabetes in NOD-CD40L $\mathrm{L}^{-/-}$mice. Taken together, our results suggest a differential role for CD28 and CD40L in controlling autoreactive $\mathrm{T}$ cell activation versus immune regulation. Moreover, a defect in Treg's can bypass the need for the two major $\mathrm{T}$ cell costimulatory pathways to facilitate the development of diabetes in individuals prone to autoimmunity, thus providing a potentially critical target of immunotherapeutic intervention.

\section{Results}

B7-2 deficiency compromises the activation of diabetogenic $T$ cells. We have previously shown that diabetes is completely abrogated in NOD-B7-2/- mice (6). In this study we examined the cellular mechanisms affected by B7-2 deficiency. To assess the activation of pathogenic $T$ cells in vivo, we analyzed the ability of autoreactive $\mathrm{T}$ cells from NOD-BDC2.5 T cell receptor (TCR) transgenic (Tg) mice, which carry a highly diabetogenic $\mathrm{T}$ cell receptor (19), to be activated by islet autoantigens in a B7-2-deficient environment. We purified $\mathrm{CD}^{+}{ }^{+} \mathrm{CD} 62 \mathrm{~L}^{+} \mathrm{CD} 25^{-} \mathrm{T}$ cells from NOD-BDC2.5 Tg mice and adoptively transferred 5-(and-6)-carboxyfluorescein diacetate, succinimidyl ester-labeled (CFSE-labeled) cells into NOD or NOD-B7-2/- recipients. The proliferation of diabetogenic BDC2.5 $\mathrm{T}$ cells was compared 2 weeks after transfer. As previously reported (2), BDC2.5 T cells primarily divided in the pancreatic LNs of NOD mice (Figure 1A, upper left) but only marginally divided in the peripheral LNs (Figure 1A, lower left) and spleen (data not shown), which is consistent with the suggestion that initial islet antigen presentation occurs in the pancreatic LNs of NOD mice (2). In contrast, proliferation of BDC2.5 T cells was greatly reduced in the pancreatic LNs of NOD-B7-2-/- mice (Figure 1, A and B) (Student's $t$ test, $P<1 \times 10^{-8}$ ). In fact, an analysis was done 30 days after transfer of BDC2.5 cells as well. In this study, the percentage of cycled cells in NOD-B7-2-/- recipient was $17 \%$, similar to what was found 2 weeks after transfer, as described above. By contrast, BDC2.5 cells had divided intensively in NOD mice to the point where CFSE was not detectable (data not shown). However, the total number of BDC2.5 cells recovered from NOD and NODB7-2-/- mice was not significantly different in either the pancreatic LNs (Figure 1C) or the peripheral LNs (data not shown). These results demonstrate that cell activation and cell cycle progression were dependent on B7-2, while homing and/or survival were not affected by B7-2 deficiency. In addition, transfer of diabetic
A
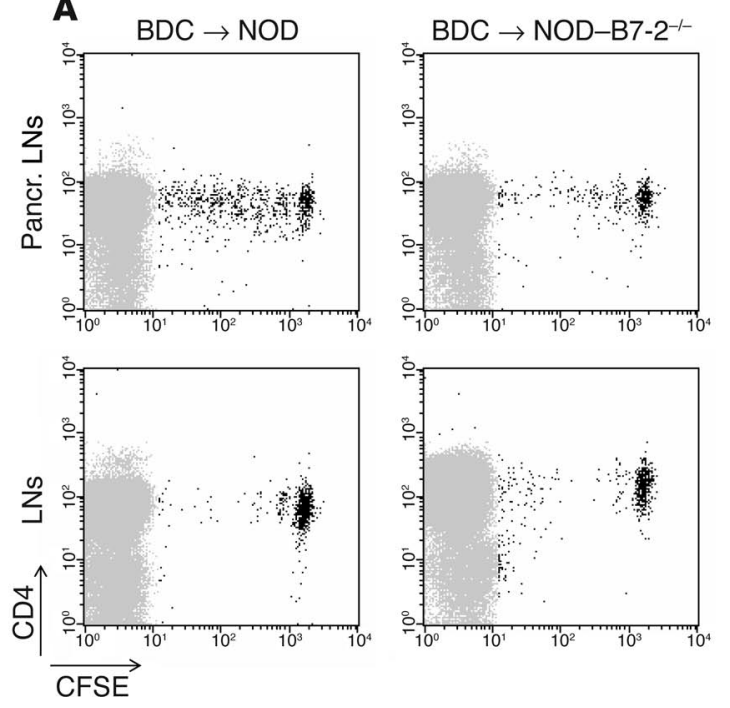

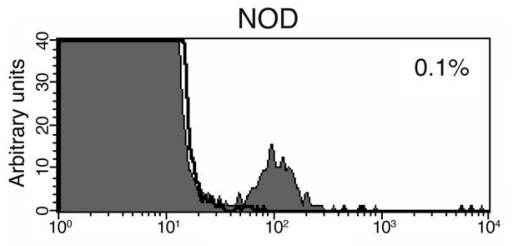

Pancreas

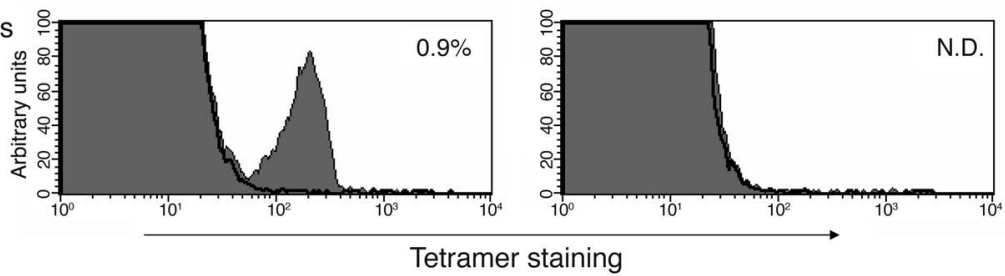

B
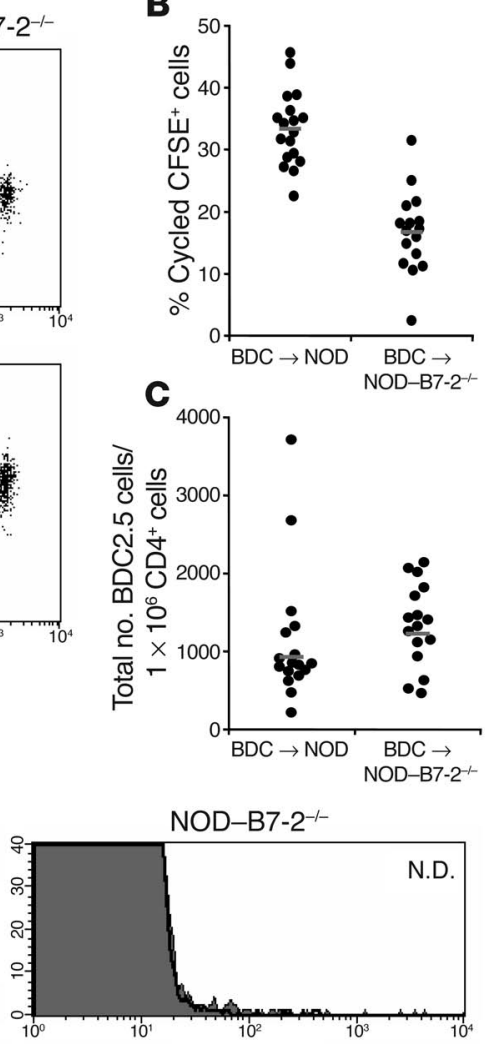

N.D.
Figure 1

B7-2 deficiency alters the initial activation of diabetogenic T cells. (A) We sorted BDC2.5 T cells, labeled them with CFSE, and transferred $1 \times 10^{6}$ cells into NOD (left panels) or NOD-B7-2-/(right panels) recipients. After 2 weeks, we examined BDC2.5 T cell proliferation in pancreatic LNs (top panels) and peripheral LNs (bottom panels). Similar results were observed in peripheral LNs and spleen (data not shown). A representative experiment is shown. Pancr., pancreatic. (B) The results of 5 separate experiments performed as described in $\mathbf{A}$ are shown. We expressed the results as the percentage of cycled $\mathrm{CFSE}^{+}$cells in the pancreatic LNs calculated as: (number of cycled CFSE ${ }^{+}$cells/total number of cycled and noncycled $\mathrm{CFSE}^{+}$cells) $\times$ 100. Each circle represents an individual mouse. Horizontal bars represent the mean value for each group. (C) We compared the total number of BDC2.5 CFSE+ cells (normalized to the number of endogenous CD4+ cells) recovered in the pancreatic LNs in NOD $(n=17)$ and NOD-B7-2-/$(n=16)$ recipients. Each circle represents an individual mouse. Horizontal bars represent the geometric mean. There was no statistical difference in the total number of BDC2.5 cells in the pancreatic LNs in NOD and NOD-B7-2-/- mice ( $t$ test using geometric mean, $P>0.05$ ). Similar results were observed in peripheral LNs (data not shown). (D) Single-cell suspensions from pancreatic LNs and pancreas of 16- to 18-weekold NOD and NOD-B7-2-/- mice were stained with CD8 and NRP-V7-H-2Kd tetramers. Results display NRP-V7-H-2Kd tetramer (filled histogram) and TUM-H-2 $\mathrm{K}^{\mathrm{d}}$ control tetramer (bold line) staining. The percentage of $\mathrm{CD} 8^{+}$tetramer $^{+}$ cells within the mononuclear cell population was indicated. N.D., not detected. 

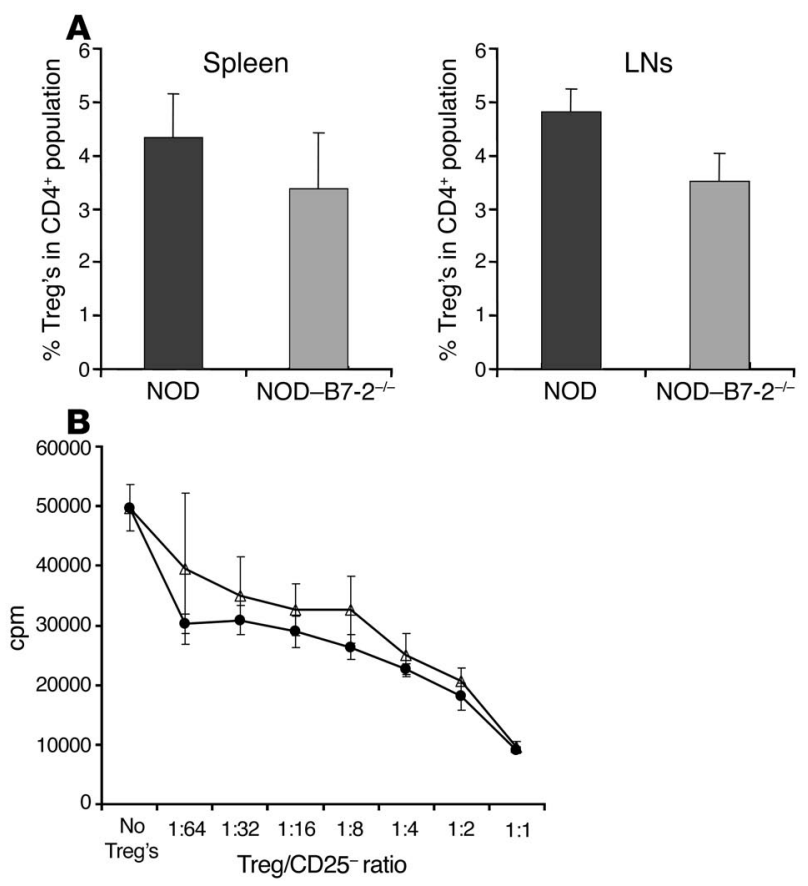

NOD T cells into NOD-RAG ${ }^{-/}$and NOD-RAG ${ }^{-/-B 7-2-/-}$ recipients induced diabetes with similar kinetics (Supplemental Figure 1; supplemental material available at http://www.jci.org/cgi/content/full/114/7/979/DC1), which suggests that B7-2 was not necessary for the effector phase of disease.

The recent identification of a prevalent population of pathogenic $\mathrm{CD}^{+} \mathrm{T}$ cells in NOD mice (20) enabled an examination of the activation of endogenous pathogenic $T$ cells in the setting of the B7-2 deficiency. $\mathrm{CD}^{+} \mathrm{T}$ cells specific for islet-specific glucose6-phosphatase catalytic subunit-related protein (IGRP) could be readily detected using tetramer technology in the pancreatic LNs and the pancreas of 16- to 18-week-old NOD mice (Figure 1D and Supplemental Figure 2, left panels). This population of pathogenic $\mathrm{CD}^{+} \mathrm{T}$ cells was undetectable in the pancreatic LNs and the pancreas of age-matched NOD-B7-2/- mice (Figure 1D and Supplemental Figure 2, right panels). Thus, the results suggest that B7-2

\section{Figure 3}

Blocking B7-1 but not CTLA-4 restores diabetes in NOD-B7-2-/- mice. (A) We treated NOD-B7-2 $2^{--}$mice with control Ig (triangles; $n=5$ ), anti-B7-1 mAb's (circles; $n=11$ ), or anti-CTLA-4 mAb's (squares; $n=8$ ). We followed blood glucose levels weekly after mice reached 8 weeks of age to assess the development of diabetes. Similar results were observed in males and females and were pooled. The incidence of diabetes was $0 \%$ in both control Ig and anti-CTLA-4 mAb-treated groups. (B) NOD-B7-2-/- mice were treated with anti-B7-1 mAb's between 2 and 4 weeks of age. Percentage of Treg's was analyzed 2-4 days after the end of the treatment. (C) BDC2.5 cells labeled with CFSE were transferred into adult (8- to 10-week-old) NOD mice or NOD-B7-2 ${ }^{-1-}$ mice treated with control Ig or anti-B7-1 mAb's. NOD$\mathrm{B} 7-2^{-1-}$ mice were treated with 7 injections of $50 \mu \mathrm{g}$ anti-B7-1 mAb's every other day using two different regimens (see Methods for details). The 2 groups gave similar results and were pooled. The results are expressed as the percentage of cycled BDC2.5 cells in the pancreatic LNs (see Figure 1 legend). Histograms represent the mean and standard deviation for each group.

\section{Figure 2}

Normal Treg function in NOD-B7-2-/- mice. (A) NOD-B7-2-/- mice have reduced percentage of Treg. We analyzed by flow cytometry the percentage of CD25+CD62L ${ }^{\text {hiTreg }}$ within the CD4+ population in NOD spleen $(n=13)$ and LNs $(n=11)$ and NOD-B7-2-/- spleen $(n=11)$ and LNs $(n=9)$. The percentage of Treg's was slightly but significantly reduced in NOD-B7-2-- mice ( $t$ test, $P=0.01$ in spleen and $P<0.00001$ in $L N s$ ). (B) NOD and NOD-B7-2-l- Treg's suppress with similar efficiency in vitro. We purified CD4 ${ }^{+} \mathrm{CD} 25^{+} \mathrm{CD} 62 \mathrm{~L}^{\text {hi }}$ Treg's from spleen and $\mathrm{LNs}$ of NOD (open triangles) and NOD-B7-2-/- (filled circles) mice. Decreasing numbers of Treg's were added to CD4 ${ }^{+} \mathrm{CD} 25-\mathrm{CD} 62 \mathrm{~L}^{\text {hi }} \mathrm{T}$ cells stimulated with anti-CD3 mAb's and APCs, and proliferation was measured by incorporation of ${ }^{3} \mathrm{H}$-thymidine.

deficiency alters the activation and expansion of both $\mathrm{CD}^{+}$and $\mathrm{CD}^{+}$diabetogenic $\mathrm{T}$ cells.

Treg's are minimally affected in NOD-B7-2-/- mice. Since CD28 is important for the homeostasis of Treg's $(14,17)$, we examined whether the Treg compartment was affected in NOD-B7-2-/mice. We focused our study on the $\mathrm{CD} 4^{+} \mathrm{CD} 25^{+} \mathrm{CD} 62 \mathrm{~L}^{\text {hi }}$ Treg subset, since cells expressing high levels of CD62L seem to have the best regulatory activity in controlling autoimmune diabetes $(21,22)$. The disruption of CD28-B7-2 interactions resulted in a slight reduction in the number of Treg's in NOD-B7-2-/- mice compared with age-matched NOD mice (Figure 2A). Indeed, Treg's were reduced from $4.3 \%$ of $\mathrm{CD}^{+} \mathrm{T}$ cells in NOD spleen to $3.4 \%$ in NOD-B7-2-/- mice (20\% reduction; Student's $t$ test, $P=0.01$ ) and from $4.8 \%$ in NOD LNs to $3.5 \%$ in NOD-B7-2-/- mice (27\% reduction; Student's $t$ test, $P<0.00001)$.

The function of Treg's from NOD-B7-2-/- mice was examined in vitro. $\mathrm{CD} 4{ }^{+} \mathrm{CD} 25^{+} \mathrm{CD} 62 \mathrm{~L}^{\text {hi }}$ Treg's isolated by FACS sorting from NOD and NOD-B7-2-/- mice had a comparable ability to suppress anti-CD3-induced proliferation on a cell-per-cell basis in vitro (Figure $2 \mathrm{~B}$ ). Thus, the number of Treg's in NOD-B7-2-/- mice was minimally affected, and the regulatory cells functioned normally.

B7-1 blockade restores diabetes in NOD-B7-2-/- mice by a Treg-dependent mechanism. Our data implied that although the costimulation provided by B7-1 was sufficient to allow the development and function of Treg's, B7-1 could not compensate for the dependence
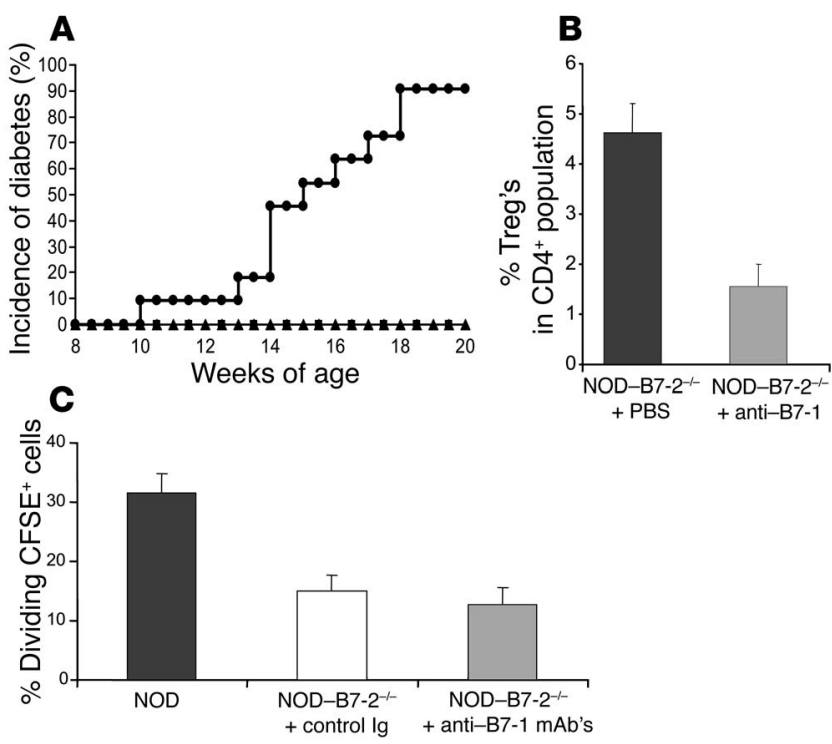

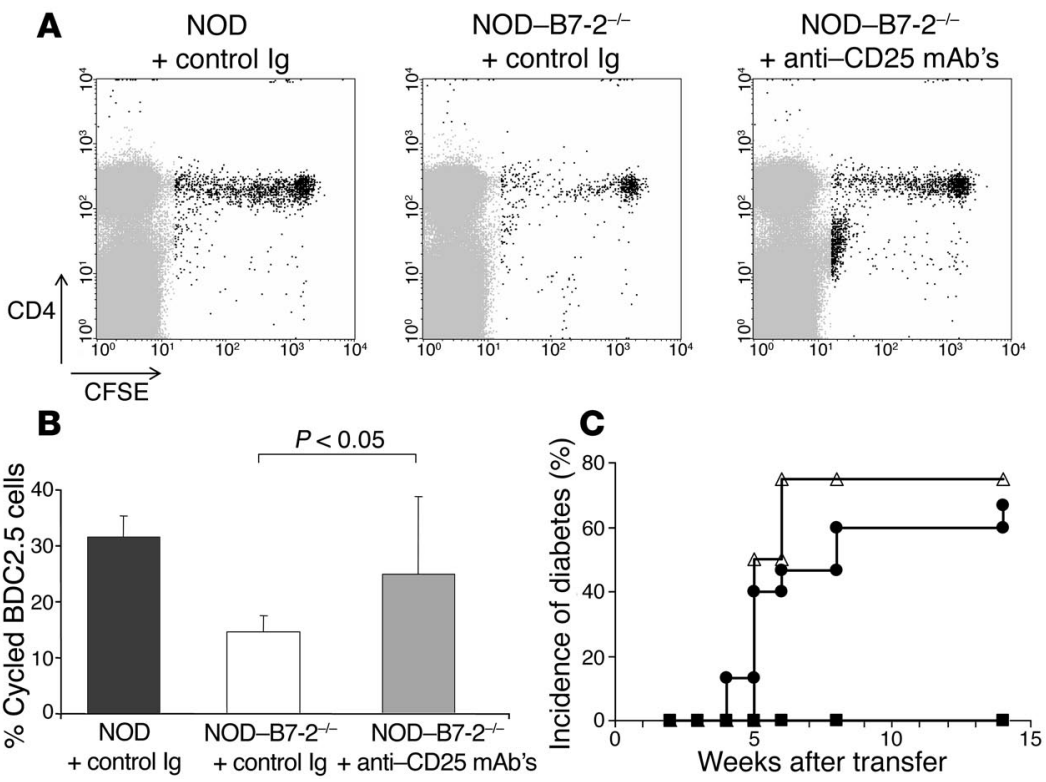

Figure 4

Treg's suppress autoreactive T cell activation in NOD-B7-2-/- mice. NOD-B7-2-/- recipients were thymectomized to avoid rapid repopulation of the peripheral Treg compartment by $\mathrm{CD} 4{ }^{+} \mathrm{CD} 25^{+}$Treg's emigrating from the thymus (46) and then treated with anti-CD25 mAb's to deplete Treg's. Moreover, the adoptive transfer of purified naive BDC2.5 T cells was delayed 2 weeks after anti-CD25 treatment to allow the antibody to clear from the circulation. (A) NOD mice were treated with control lg (left) and NOD-B7-2-1- mice were treated with control Ig (middle) or anti-CD25 mAb's (PC61) (right). Transfer of BDC2.5 cells and analysis in the pancreatic LNs was as described in Figure $1 \mathrm{~A}$. A representative experiment is shown. (B) The results of 3 separate experiments performed as described in $\mathbf{A}$ are shown. Percentage of cycled $\mathrm{CFSE}^{+}$cells in the pancreatic $\mathrm{LNs}$ was calculated as in Figure 1B in NOD, NOD-B7-2-/-, and NOD-B7-2-/- depleted of Treg's. Each histogram represents the mean and standard deviation of each group. The $P$ value for the $t$ test between the relevant groups is shown. The same result was obtained when comparing geometric means instead of means $(t$ test, $P<0.05)$. (C) We depleted Treg's from NOD or NOD-B7-2 $2^{--}$spleen cells using anti-CD25 mAb's (7D4) and rabbit complement. We transferred total spleen cells from NOD-B7-2-1- mice (filled squares; $n=5$ ) or CD25depleted spleen cells from NOD mice (open triangles; $n=4$ ) or NOD-B7-2-1- mice (filled circles; $n=9$ ) into NOD-SCID recipients. We followed blood glucose levels weekly after transfer to assess the development of diabetes.

of B7-2 in the activation of pathogenic T cells in NOD mice. This result suggested that there might be differential expression of B7-1 and B7-2 in the relevant antigen-presenting tissues. Therefore, we analyzed the expression of B7-1 in the pancreatic LNs. B7-1 was not detectable in the pancreatic LNs of NOD-B7-2/- mice (data not shown). By comparison, as shown previously, B7-1 was constitutively expressed in the spleen of NOD-B7-2/- mice as compared with NOD mice (6). Moreover, in contrast, B7-1 expression was increased in the pancreatic LNs compared with the spleen of wild-type NOD mice (data not shown). Thus, the low expression of B7-1 in the pancreatic LNs likely was responsible for the defect in pathogenic $T$ cell activation in NOD-B7-2-- mice. Interestingly, treatment of 2- to 4-week-old NOD-B7-2-- mice with anti-B7-1 mAb's resulted in the development of diabetes in these otherwise protected animals (Figure $3 \mathrm{~A}$ ). Indeed, $90 \%$ of NOD-B7-2/- mice treated with anti-B7-1 became diabetic by 18 weeks of age. This result suggested that a diabetogenic repertoire was present in NOD-B7-2/- mice but was regulated "negatively" by a B7-1-dependent mechanism such as the development of Treg's or the engagement of CTLA- 4 .
To address whether the effect of anti-B7-1 was related to Treg activity, we examined anti-B7-1treated mice for Treg's over a 2-week period. As seen in Figure 3B, the number of Treg's decreased dramatically to $1.5 \%$ of the $\mathrm{CD} 4^{+} \mathrm{T}$ cell subset compared with $4.6 \%$ in the control group during the treatment. This result is in agreement with our recent reports that $\mathrm{CD} 28$ controls homeostasis of Treg's $(14,17)$ and further suggests that B7-1 is necessary and sufficient for the development of a normal Treg compartment in NOD-B7-2-/- mice. Moreover, treatment of NOD-B7-2 $2^{-/}$mice with anti-CTLA-4 mAb's using a well-established protocol $(23,24)$ had no effect on disease incidence despite the fact that mice were treated at various stages of disease progression (2-7 weeks of age) (Figure $3 \mathrm{~A}$ ). These results suggest that induction of diabetes following the blockade of B7-1 was due to elimination of Treg's, not CTLA-4 function.

The increase in diabetes following blockade of B7-1 in NOD-B7-2-/- mice suggested that CD28 costimulation was dispensable for the activation of autoreactive $\mathrm{T}$ cells. We thus analyzed whether B7-1 blockade would affect the activation of BDC2.5 cells in the pancreatic LNs of NOD-B7-2/- mice. We treated adult NOD-B7-2-/- mice with anti-B7-1 $\mathrm{mAb}$ 's and transferred BDC2.5 cells labeled with CFSE. As shown in Figure 3C, the proliferation of BDC2.5 cells was unaffected 2 weeks after transfer, although the percentage of $\mathrm{CD} 4^{+} \mathrm{CD} 25^{+}$Treg's was greatly decreased following B7-1 blockade ( $0.5 \%$ Treg's in anti-B7-1-treated mice compared with $2.8 \%$ in mice treated with control Ig).

Treg's control activation of diabetogenic $T$ cells in NOD $-B 7-2^{-/-}$mice. Our results suggested that $\mathrm{CD} 4^{+} \mathrm{CD} 25^{+}$Treg's could control a potentially pathogenic repertoire in NOD-B7-2/- mice. To directly test this hypothesis, we designed an experimental protocol to deplete Treg's without affecting potentially pathogenic $\mathrm{T}$ cells that had upregulated CD25 as a consequence of antigen activation. Complete depletion of Treg's was achieved by antiCD25 (PC61) treatment at the time of transfer of BDC2.5 T cells: $0.3 \%$ Treg's compared with $2.4 \%$ in animals treated with control Ig (Supplemental Figure 3A). The number of Treg's was still diminished 2 weeks after transfer of BDC2.5 cells in anti-CD25-treated mice $1.9 \%$ Treg's compared with $3.5 \%$ in animals treated with control Ig) (Supplemental Figure 3B). As observed in euthymic mice (Figure 1), proliferation of BDC2.5 T cells in the pancreatic LNs was greatly reduced in thymectomized control Ig-treated NOD-B7-2 $2^{--}$mice as compared with NOD mice (Figure 4A). In contrast, depletion of Treg's partially restored proliferation of $\mathrm{BDC} 2.5 \mathrm{~T}$ cells in the pancreatic LNs of NOD-B7-2/- mice (Figure $4 \mathrm{~A})$. Indeed, the percentage of divided cells within the BDC2.5 T cell population significantly increased after PC61 treatment compared with control mAb's (Student's $t$ test, $P<0.05$ ) (Figure 4B). BDC2.5 $\mathrm{T}$ cell proliferation in the Treg-deficient setting occurred in the pancreatic LNs and not in other LNs (Supplemental Figure 3C), which suggests that depletion of Treg's resulted in islet antigen-specific activation of diabetogenic $\mathrm{T}$ cells in the pancreatic 
A
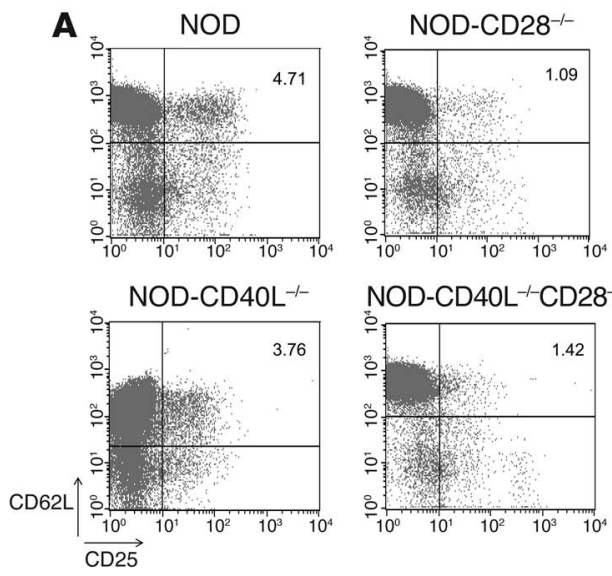

NOD-CD40L ${ }^{-/-} \mathrm{CD} 28^{-/-}$
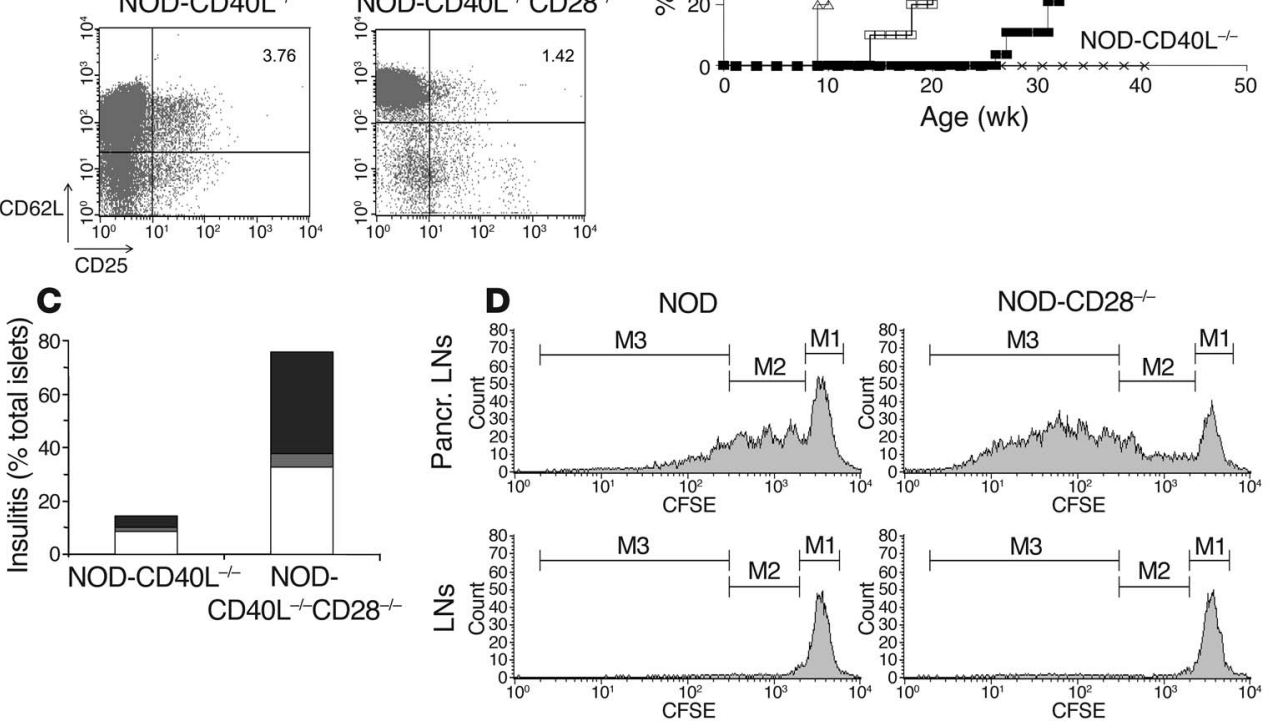

Figure 5

CD28 and CD40L differentially affect diabetes in NOD mice. (A) We developed NOD mice deficient for CD28 (NOD-CD28---), CD40L (NOD-CD40L ${ }^{-/}$), or both CD40L and CD28 (NOD-CD40L ${ }^{-/-} \mathrm{CD}^{-1} 8^{-/}$). We compared the number of Treg's in these different strains. The results are displayed as CD62L ( $y$ axis) versus CD25 ( $x$ axis) staining and are gated on the CD4+ population. One out of 3 representative experiments is shown. (B) We compared the incidence of diabetes in NOD (open squares), NOD-CD28 ${ }^{-1-}$ (open triangles), NOD-CD40L $\mathrm{L}^{-/}$(X's) and NOD-CD40L ${ }^{-1-} \mathrm{CD}_{28} 8^{--}$(filled squares) mice. (C) We performed histological analysis of the pancreas in NOD-CD40L ${ }^{-/-}$and NOD-CD40L ${ }^{-1} \mathrm{CD} 28^{-/-}$mice and scored the severity of insulitis as peri-insulitis (white bars), moderate insulitis (gray bars) and severe insulitis (black bars) in NOD-CD40L-/and NOD-CD40L ${ }^{-1-C D 28}-/-$ mice (70 and 37 islets were scored, respectively). (D) CD25-depleted BDC2.5CD90.1 T cells were labeled with CFSE and transferred into NOD or NOD-CD28 $8^{-1-}$ mice. Four days later, the proliferation of BDC2.5 cells was assessed in the pancreatic LNs. The histograms are gated on CD4+ CD90.1+ cells, and the 3 regions M1-M3 indicate undivided cells, cells that underwent 1 to 3 divisions, and cells that underwent 4 or more divisions, respectively.

LNs rather than nonspecific polyclonal activation in all peripheral lymphoid tissues. In addition, 1 of 9 NOD-B7-2-/- recipients treated with anti-CD25 mAb's and engrafted with BDC2.5 T cells became diabetic within 2 weeks (data not shown), which further underscores the dominant role of $\mathrm{CD} 4{ }^{+} \mathrm{CD} 25^{+}$Treg's in controlling autoimmune diabetes in NOD-B7-2/- mice.

To analyze whether potentially pathogenic polyclonal $\mathrm{T}$ cells were being similarly suppressed in NOD-B7-2-/- mice, we depleted $\mathrm{CD} 25^{+} \mathrm{T}$ cells from NOD-B7-2-/- spleen cells and transferred the remaining cells into NOD-SCID mice. Whole spleen cells from NOD-B7-2/- mice failed to transfer diabetes into NOD-SCID recipients (Figure $4 \mathrm{C}$ ). In sharp contrast, depletion of $\mathrm{CD} 25^{+}$ Treg's from NOD-B7-2-/- spleen cells prior to transfer resulted in development of diabetes in $70 \%$ of the recipients, with an incidence and kinetics of disease similar to what was observed using CD25depleted spleen cells from NOD mice (Figure 4C). Taken together, these results suggest that $\mathrm{CD} 4{ }^{+} \mathrm{CD} 25^{+}$Treg's efficiently suppressed potentially diabetogenic BDC2.5 T cells in the pancreatic
LNs of NOD-B7-2-/- mice. Thus, although it has been suggested that CD28 could play a role in thymic selection (25-28), a diabetogenic repertoire was present in NOD-B7-2-/- mice but efficiently suppressed by resident Treg's. Moreover, these results suggested that Treg's actively suppressed rather than tolerized pathogenic $\mathrm{T}$ cells in NOD-B7-2-/- mice.

CD28 and CD40L differentially influence autoreactivity and regulation. The previous experiments suggested that although CD28B7-2 interactions played an important role in the activation of naive islet antigen-specific $\mathrm{T}$ cells, the presence of CD28dependent $\mathrm{CD} 4^{+} \mathrm{CD} 25^{+}$Treg's was the dominant mechanism preventing manifestation of the disease. Since CD40L interactions with CD40 have been described as the other major costimulatory pathway for diabetogenic $T$ cells in NOD mice (7-9), we examined how disrupting CD28-dependent Treg's would affect diabetes in the absence of CD40L-CD40 interactions. NOD-CD28 $8^{-/}$, NOD-CD $40 \mathrm{~L}^{-/-}$and NOD mice deficient for both CD40L and CD28 (NOD-CD40L-/-CD28-/-) were compared for Treg numbers and diabetes incidence. Similarly to NOD-B7-2-/- mice, NODCD40L $\mathrm{L}^{-/-}$mice displayed significant yet moderate reduction in Treg numbers as compared with NOD (Figure 5A). In addition, NOD-CD40L $\mathrm{L}^{-/-} \mathrm{CD} 28^{-/-}$mice displayed a dramatic decrease in Treg's as compared with NOD and NOD-CD40L ${ }^{-/-}$mice (Figure 5A).

We found that, in agreement with what has been shown previously $(8,9)$, NOD-CD $40 \mathrm{~L}^{-/-}$mice did not develop diabetes. In contrast, the incidence of diabetes was partially restored in NOD-CD40L $-/-$ CD28 $/ /-$ mice compared with NOD-CD40L $\mathrm{L}^{-/-}$(Figure 5B), although with delayed kinetics. Histological analysis further demonstrated that whereas infiltration was virtually absent in the pancreatic islets of NOD-CD $40 \mathrm{~L}^{-/-}$mice, severe insulitis was observed in NOD-

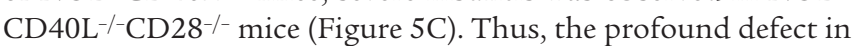
Treg's observed in NOD-CD40L $\mathrm{L}^{-/-} \mathrm{CD} 28^{-/-}$mice partially bypassed the need for either CD40L or CD28 costimulation in pathogenic $T$ cell activation and development of autoimmunity toward the pancreatic islets. However, incidence of diabetes was lower in NOD-CD $40 \mathrm{~L}^{-/-} \mathrm{CD} 28^{-/-}$mice compared with NOD-CD $28^{-/-}$ mice, which suggests that severity of disease was affected by the relative decrease in pathogenic $\mathrm{T}$ cell activation due to defective CD40L costimulation. 

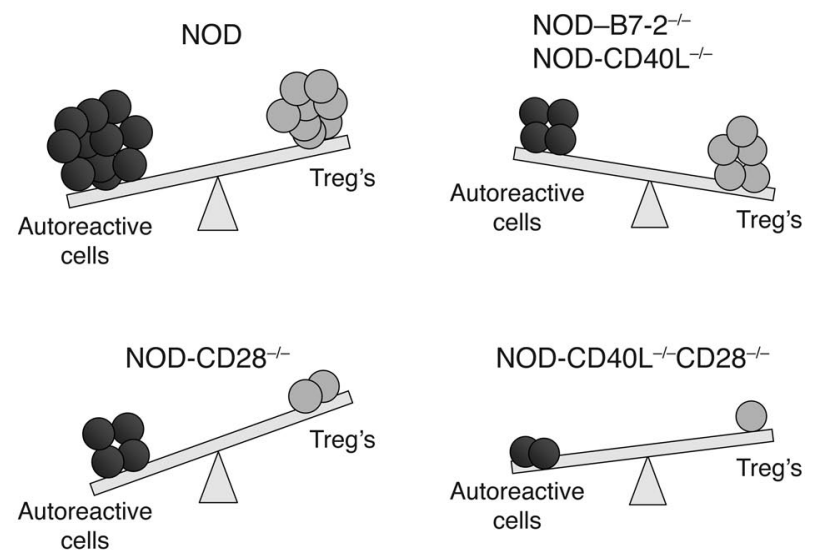

Figure 6

Autoimmune diabetes is controlled by a costimulation-dependent balance of pathogenic and regulatory T cells. In NOD mice, which are genetically prone to autoimmunity, autoreactive T cells dominate Treg's, leading to development of autoimmune diabetes. In the absence of B7-2 or CD40L, Treg's are marginally reduced, whereas activation of autoreactive $T$ cells is profoundly affected, resulting in the absence of diabetes. In contrast, in the absence of CD28 signals, the deficit in Treg's is so severe that disease develops with accelerated kinetics and increased severity. Finally, in the absence of CD28 and CD40L signals, both diabetogenic T cells and Treg's are affected, but the absence of CD28-dependent Treg's appears dominant and results in insulitis and diabetes, thus bypassing the need for the two major costimulatory pathways in the development of the autoimmune process.

To directly assess how a defect in Treg's affected the autoreactive population, we transferred CFSE-labeled CD25-depleted BDC2.5 $\mathrm{T}$ cells into NOD and NOD-CD28-/- mice and compared the proliferation of the autoreactive cells in the pancreatic LNs. The expansion of BDC2.5 T cells was dramatically accelerated in NODCD28 $/ /$ mice as compared with NOD mice (Figure 5D). Indeed, the number of undivided cells and cells that underwent 1 to 3 divisions was similar in NOD and NOD-CD28 $8^{-/}$, but the number of cells that underwent 4 or more divisions was on average 8 times higher in NOD-CD28-/- compared with NOD pancreatic LNs (Figure 5D and Supplemental Figure 4A). In addition, the enumeration of BDC2.5 T cells revealed that NOD-CD28 $8^{--}$contained 5 times more autoreactive BDC2.5 T cells in the pancreatic LNs compared with NOD mice (Supplemental Figure 4B). Finally, the increased division of autoreactive cells observed in NOD-CD28-/mice was specifically driven by pancreatic islet antigens in the draining pancreatic LNs, since no proliferation was observed in other peripheral LNs (Figure 5D and Supplemental Figure 4A).

\section{Discussion}

It has become increasingly clear that a delicate balance of pathogenic and protective immune responses controls the development and clinical manifestation of type I diabetes. Although CD28-B7 and CD40L-CD40 interactions have been long recognized as the two major costimulatory pathways of $\mathrm{T}$ cell responses, the potential control of immunoregulatory mechanisms by costimulatory molecules is only starting to be unraveled. In this study, we showed that B7-2 was necessary for the optimal activation of diabetogenic T cells in the pancreatic LNs but not for the development of a functional Treg compartment. In addition, affecting the Treg compartment by complete disruption of CD28 signals or deple- tion of $\mathrm{CD} 4^{+} \mathrm{CD} 25^{+} \mathrm{T}$ cells restored pathogenic $\mathrm{T}$ cell activation and diabetes. Finally, Treg's were reduced and insulitis and dia-

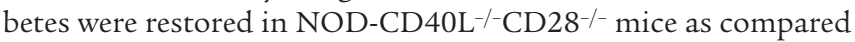
with NOD-CD40L $\mathrm{L}^{-/-}$mice. These results suggest that CD28-B7-2 and CD40L-CD40 interactions play a predominant role in diabetogenic $\mathrm{T}$ cell activation, whereas complete blockade of CD28 costimulation severely impacts the Treg compartment. Thus, a deficient Treg compartment circumvents the need for the two major costimulatory pathways to achieve autoimmunity against pancreatic islets. In this setting, costimulation requirements could be modified by chronic presentation of autoantigen (29). Alternatively, costimulatory molecules such as inducible costimulator (ICOS), OX40 (CD134), or other as-yet-unidentified costimulatory molecules could play a role in $\mathrm{T}$ cell activation in the absence of CD28 and CD40L. In sum, diabetes is controlled by a balance of pathogenic and regulatory $\mathrm{T}$ cells, and although costimulatory signals regulate both arms of the balance, CD28-dependent Treg's appear to be the dominant factor in the development of the autoimmune process (Figure 6).

The number of Treg's in NOD-B7-2-/- mice was slightly reduced as compared with NOD mice, and blocking B7-1 interactions with CD28 in NOD-B7-2/- mice decreased the number of Treg's to levels similar to those in NOD-CD28 $/ /$ mice. These results suggest that B7-1 and B7-2 additively signal through CD28 to maintain Treg homeostasis and that B7-1 is sufficient to maintain a functional Treg compartment in NOD mice. In addition, the development of diabetes in NOD-B7-2/- mice treated with anti-B7-1 mAb's demonstrates that low levels of constitutive B7 expression play a prominent role in maintaining peripheral tolerance and preventing autoimmunity. This result confirms our previous report that blocking the CD28-B7 pathway in NOD breaks peripheral tolerance and results in exacerbated diabetes $(14,18)$. Recently, Lohr et al. showed that basal B7 expression on APCs reduced T cell responses in vivo and that $\mathrm{CD} 4{ }^{+} \mathrm{CD} 25^{+}$Treg's were responsible for this effect (30). In addition, low levels of B7-1 expression in the pancreatic LNs compared with the spleen of NOD-B7-2/- mice suggest that CD28 signals provided in other tissues is enough to maintain a functional Treg compartment in the LNs.

An alternative explanation for the pro-diabetogenic effects of blocking B7-1 in NOD-B7-2-/- mice is that this treatment prevents CTLA-4 ligation and results in increased activation of autoreactive T cells. However, treatment of NOD-B7-2/- mice with anti-CTLA-4 failed to reproduce the increase in diabetes secondary to anti-B7-1 blockade, which suggests that the effects of this treatment are not limited to disruption of CTLA-4 engagement. Moreover, CTLA-4 has been shown to be most effective in controlling secondary rather than naive responses (31) that are clearly defective in this model. In this regard, our data showed that anti-B7-1 treatment was mainly effective when administered early in disease development (at 2-4 weeks of age; i.e., before the development of insulitis), whereas Mathis and colleagues demonstrated in the NOD-BDC2.5 TCR Tg model that CTLA-4 controlled autoimmune diabetes principally after insulitis was established (32). Finally, NOD mice deficient in CD40L, B7-1, and B7-2 do not develop exacerbated disease as compared with NOD-CD40L ${ }^{-/-} \mathrm{CD} 28^{-/-}$mice (data not shown), which suggests that additional disruption of CTLA-4 did not dramatically affect the balance of activation and regulation in that setting. In this regard, it has been suggested previously that B7 molecules could costimulate $T$ cell responses by interacting with ligands other than CD28 and CTLA-4 on T cells (33), and this 
could explain the increased autoimmune response in mice deficient for CD28 and CD40L compared with mice lacking CD40L and both B7-1 and B7-2.

Although it is well established that Treg's suppress effector $\mathrm{T}$ cell proliferation in vitro, the mechanism of suppression by Treg's in vivo is still controversial $(30,34-36)$. Our results suggest that Treg's control diabetogenic T cells in NOD mice by suppressing their initial islet antigen-specific activation and expansion in the pancreatic LNs and thus before they could reach later stages of differentiation or effector function. This is in agreement with several studies showing suppression of proliferation by Treg's in vitro and in vivo $(34,36-38)$. However, this result is in contrast to recent reports suggesting that Treg's do not affect proliferation but rather production of IFN- $\gamma$ in vivo $(30,35,39)$. In addition, Herman and colleagues recently showed that $\mathrm{CD} 4^{+} \mathrm{CD} 25^{+} \mathrm{T}$ cells expressed the Treg-specific transcription factor Foxp 3 and regulated diabetogenic $T$ cells directly in the pancreas (40). This discrepancy could be explained by the different experimental systems used. In spontaneous autoimmune models, low-affinity autoreactive $T$ cells are activated by their natural autoantigen in the pancreatic LNs. This is in contrast with other TCR Tg models, where higher-precursor-frequency and high-affinity $\mathrm{T}$ cells are examined or where activation is maximized by dendritic cells loaded with foreign antigen $(30,35,39)$. Thus, our results suggest that Treg's may suppress primary $\mathrm{T}$ cell activation in vivo, especially under conditions of low-avidity TCR interactions due to low-affinity $\mathrm{T}$ cell receptors and/or chronic presentation of low levels of antigens such as autoantigens.

Depletion of $\mathrm{CD}^{+} \mathrm{CD} 25^{+}$Treg's partially restored the proliferation of islet antigen-specific T cells in the pancreatic LNs of NOD-B7-2/- mice and promoted diabetes in an adoptive transfer setting. Similarly, the defect in Treg's in NOD-CD $28^{-/-}$mice resulted in accelerated disease and increased number and expansion of autoreactive $\mathrm{T}$ cells in the pancreatic LNs compared with that in NOD mice. Finally, whereas it is known that NOD-CD40L-/- mice fail to develop diabetes due to inefficient activation of autoreactive T cells $(7,9)$, our data showed that insulitis and diabetes were partially restored in NOD-CD $40 \mathrm{~L}^{-/-} \mathrm{CD} 28^{-/-}$mice as a result of decreased Treg numbers. Although it has been shown that the CD40L-CD40 interaction plays a role in Treg development (41), our results strongly suggest that the Treg compartment in NOD mice is principally controlled by the CD28-B7 pathway. This is in agreement with the recent report that disruption of CD40L-CD40 interactions affected helper T cells but not Treg's in NOD mice (42). Moreover, enhanced autoimmunity in NOD-CD40L $\mathrm{L}^{-/-} \mathrm{CD} 28^{-/-}$ mice compared with NOD-CD40L-/- mice reveals that CD28 and CD40L control autoimmunity via distinct pathways. This is in contrast to the hypothesis that defective CD40 ligation affects $\mathrm{T}$ cell responses indirectly due to impaired upregulation of $\mathrm{B} 7$ molecules leading to insufficient CD28 costimulation $(10,11)$. The observation that depletion of $\mathrm{CD} 4^{+} \mathrm{CD} 25^{+}$Treg's only partially restored the proliferation of BDC2.5 T cells and/or the development of diabetes could be due to limiting costimulation leading to reduced activation of pathogenic T cells. Similarly, the absence of BDC2.5 cell proliferation following anti-B7-1 treatment in adult NOD-B7-2-/mice could reflect the absence of CD28 costimulation, resulting in delayed $\mathrm{T}$ cell activation that could not take place within the 2-week period of our experiment. Alternatively, other Treg populations such as NKT cells $(43,44)$ and $\mathrm{DX}^{+} \mathrm{T}$ cells $(45)$ have been shown to protect NOD mice from diabetes and could be playing a regulatory role in the absence of $\mathrm{CD} 4^{+} \mathrm{CD} 25^{+}$Treg's. Finally, it is possible that costimulation-regulated Treg's could function in an age-dependent manner in NOD mice, which would thus explain the differential efficiency of B7-1 blockade observed in 2- to 4-weekold versus adult mice.

Taken together, our results have several important implications. First, the development of diabetes in NOD mice deficient for both CD40L and CD28 demonstrates that Treg's play a dominant role in the balance of autoreactivity versus regulation that determines the outcome of disease. Second, in the absence of a functional Treg compartment, the two major costimulatory pathways can be bypassed for pathogenic $\mathrm{T}$ cell activation in individuals prone to autoimmunity. Third, while both CD28-B7-2 and CD40LCD40 interactions controlled the activation of pathogenic $\mathrm{T}$ cells, constitutive levels of CD28-B7 interactions played a unique role in maintaining an efficient Treg compartment. Therapeutic approaches could thus be designed to affect selectively CD28-B7-2 and CD40L-CD40 interactions while maintaining a residual level of CD28 costimulation in order not to eliminate Treg's. In addition, it will be essential to establish a careful monitoring of Treg's when patients are treated with drugs blocking costimulatory pathways. In conclusion, these results have important consequences for the design of immunotherapy aimed at selectively suppressing autoimmunity and suggest that it will be essential to maximize immune regulation in addition to targeting autoreactive $T$ cells in autoimmune-prone individuals.

\section{Methods}

Mice. NOD mice were purchased from Taconic. NOD-B7-2/- and NODCD28 $8^{-/}$mice have been described previously $(6,14,18)$. NOD-BDC2.5 TCR $\mathrm{Tg}$ mice (19) were generously provided by C. Benoist and D. Mathis (Harvard Medical School, Boston, Massachusetts, USA). NOD-CD40L $/-$ mice were generously provided by R.A. Flavell (Yale University School of Medicine, New Haven, Connecticut, USA). NOD-CD28-/- mice were bred to NOD$\mathrm{CD}_{40 \mathrm{~L}} /-$ mice, and $\mathrm{F}_{1}$ heterozygous mice were intercrossed to obtain NOD-

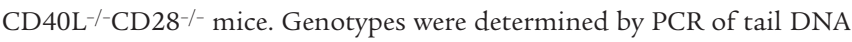
using primers specific for CD28 and CD40L. NOD-BDC2.5-CD90.1 mice were derived in our laboratory. All mice were housed in a pathogen-free facility at the University of California at San Francisco. Experiments complied with the Animal Welfare Act and the NIH guidelines for the ethical care and use of animals in biomedical research.

Assessment of diabetes and insulitis. Blood glucose levels were measured every week with a One Touch II glucose meter (Lifescan). Mice were considered diabetic after 2 consecutive measurements over $300 \mathrm{mg} / \mathrm{dl}$. For histological analysis, pancreases were snap-frozen in OCT. Multiple $5-\mu \mathrm{m}$ sections were stained with hematoxylin and eosin and scored blindly for severity of insulitis as previously described (14).

Antibodies and other reagents. Anti-CD3e (145-2C11), anti-B7-1 (16.10A1), anti-CTLA-4 (4F10), anti-CD25 (PC61), and anti-CD4 (GK1.5) mAb's were produced in our laboratory. Control rat IgG and hamster IgG were obtained from Jackson ImmunoResearch Laboratories. Anti-CD25-phycoerythrin $\mathrm{mAb}$ (7D4) was obtained from Southern Biotechnology Associates Inc. Anti-CD4-peridinin-chlorophyll-protein (RM4.5) and anti-CD62Lallophycocyanin $\mathrm{mAb}$ 's were purchased from $\mathrm{BD}-\mathrm{Pharmingen.}$ CFSE was purchased from Molecular Probes Inc. NRP-V7-H-2 $\mathrm{K}^{\mathrm{d}}$ and TUM-H- $2 \mathrm{~K}^{\mathrm{d}}$ tetramers (20) were generously provided by Pere Santamaria (University of Calgary, Calgary, Alberta, Canada).

In vivo mAb treatment. Mice were treated with $50 \mu \mathrm{g}$ anti-B7-1 mAb's every other day for 14 days at 2-4 weeks of age or with $50 \mu \mathrm{g}$ anti-CTLA- $4 \mathrm{mAb}$ 's every other day for 14 days at 2-4 weeks of age or 5-7 weeks of age, as indi- 
cated in the figure legends. Similar results were observed in mice treated at these two age-periods and were pooled. The efficiency of this treatment in vivo was well established as exacerbating ongoing immune responses in experimental autoimmune encephalitis, tumor immunity, and other immune response settings $(23,24)$. In all experiments involving $\mathrm{mAb}$ treatments in vivo, control mice received either PBS, control rat IgG, or hamster IgG at the same regimen. Similar results were observed with control IgG treatments and PBS treatments.

Cell sorting and flow cytometry. Single-cell suspensions were prepared from the spleen and LNs of indicated mice. Treg's were sorted on a MoFlo cytometer (DakoCytomation) to greater than $95 \%$ purity based on the phenotype $\mathrm{CD} 4{ }^{+} \mathrm{CD} 62 \mathrm{~L}^{\text {hi }} \mathrm{CD} 25^{+}$. Naive $\mathrm{CD} 4^{+} \mathrm{T}$ cells were sorted based on the phenotype $\mathrm{CD} 4{ }^{+} \mathrm{CD} 62 \mathrm{~L}^{\text {hi }} \mathrm{CD} 25^{-}$. Flow cytometric analyses were performed on a FACSCalibur flow cytometer with CELLQuest software (BD - Pharmingen). For tetramer staining, single-cell suspensions were prepared from pancreatic LNs and pancreas (after digestion with collagenase and DNase) from 16- to 18-week-old mice as previously described (6), and tetramer staining was performed as previously described (20).

Adoptive transfer experiments. Sorted naive BDC2.5 T cells were labeled with $1.5 \mu \mathrm{M}$ CFSE, and $1 \times 10^{6}$ cells were transferred via retro-orbital injection. In some experiments, recipient mice were thymectomized and treated by intraperitoneal injection with $500 \mu \mathrm{g}$ anti-CD25 mAb's (PC61) or control rat IgG 2 weeks prior to adoptive transfer of BDC2.5 cells. In some experiments, recipient mice were treated by intraperitoneal injection with $50 \mu \mathrm{g}$ anti-B7-1 mAb's or control hamster IgG every other day for 14 days either until 1 week before adoptive transfer of BDC2.5 cells or with the same regimen until 1 week before transfer and for 2 weeks after transfer. Similar results were observed with these two regimens and were pooled.

Ten to 28 days after adoptive transfer, BDC2.5 T cell proliferation was examined by flow cytometry by analyzing CFSE dilution within the CD $4^{+}$ $\mathrm{T}$ cell population in LNs and pancreatic LNs. The number of BDC2.5 cells that had gone into cell cycle was calculated as the number of events in each CFSE peak divided by the number of cell division corresponding to this peak (i.e., number of events divided by 2 for one division, number of events divided by 4 for two divisions, etc.). The total numbers of divided and nondivided BDC2.5 T cells were normalized to the number of endogenous $\mathrm{CD} 4^{+} \mathrm{T}$ cells.

1. Delovitch, T.L., and Singh, B. 1997. The nonobese diabetic mouse as a model of autoimmune diabetes: immune dysregulation gets the NOD. Immunity. 7:727-738.

2. Hoglund, P., et al. 1999. Initiation of autoimmune diabetes by developmentally regulated presentation of islet cell antigens in the pancreatic lymph nodes. J. Exp. Med. 189:331-339.

3. Bach, J.F. 2002. Immunotherapy of type 1 diabetes: lessons for other autoimmune diseases. Arthritis Res. 4:S3-S15.

4. Salomon, B., and Bluestone, J.A. 2001. Complexities of CD28/B7: CTLA-4 costimulatory pathways in autoimmunity and transplantation. Annu. Rev. Immunol. 19:225-252.

5. Lenschow, D.J., et al. 1995. Differential effects of antiB7-1 and anti-B7-2 monoclonal antibody treatment on the development of diabetes in the nonobese diabetic mouse. J. Exp. Med. 181:1145-1155.

6. Salomon, B., et al. 2001. Development of spontaneous autoimmune peripheral polyneuropathy in B7-2deficient NOD mice. J. Exp. Med. 194:677-684.

7. Balasa, B., et al. 1997. CD40 ligand-CD40 interactions are necessary for the initiation of insulitis and diabetes in nonobese diabetic mice. J. Immunol. 159:4620-4627.

8. Green, E.A., Wong, F.S., Eshima, K., Mora, C., and Flavell, R.A. 2000. Neonatal tumor necrosis factor alpha promotes diabetes in nonobese diabetic
Depletion of $\mathrm{CD}_{25} 5^{+}$cells from spleen and $\mathrm{LN}$ cells and adoptive transfer into NOD-SCID, NOD, or NOD-CD28 $/$ - recipients was performed as previously described (14). In some experiments, CD25-depleted BDC2.5 CD90.1 cells were labeled with CFSE as above prior to transfer, and proliferation in vivo was assessed 4 days later.

In vitro assay for Treg function. Effector $\mathrm{CD} 4^{+} \mathrm{CD} 62 \mathrm{~L}^{\text {hi }} \mathrm{CD} 25^{-} \mathrm{T}$ cells and $\mathrm{CD} 4{ }^{+} \mathrm{CD} 62 \mathrm{~L}^{\text {hi }} \mathrm{CD} 25^{+}$Treg's were sorted from indicated mice. DMEM-glutamax medium supplemented with $100 \mathrm{U} / \mathrm{ml}$ penicillin, $100 \mathrm{U} / \mathrm{ml}$ streptomycin, nonessential amino acids, $10 \mathrm{mM}$ HEPES (all from UCSF Cell Culture Facility, San Francisco, California, USA), 5\% heat-inactivated FCS (Summit Biotechnology), and $50 \mu \mathrm{M} \beta$-mercaptoethanol (Invitrogen Life Technologies) was used for cell culture. A total of $5 \times 10^{4}$ effector T cells was stimulated with $3 \mu \mathrm{g} / \mathrm{ml}$ anti-CD3 $\varepsilon$ in the presence of $5 \times 10^{4}$ irradiated APCs from NOD-TCRa ${ }^{-/}$mice. Treg's were added to the culture to obtain Treg's/naive cells ratios of 1:1 to 1:64. Proliferation was measured after 72 hours by overnight incorporation of ${ }^{3} \mathrm{H}$-thymidine (NEN; Perkin Elmer). Proliferation experiments were performed in triplicate wells.

\section{Acknowledgments}

This work was supported by research grants from the NIH (AI50834) and the Juvenile Diabetes Research Foundation (4-1999841). The authors thank P. Weghfart for animal care; C. Cusack for technical support; S. Jiang and C. McArthur for Mo-Flo sorting; A.K. Abbas, L. Chatenoud, E.L. Masteller, and Q. Tang for critical reading of the manuscript; and members of the Bluestone laboratory for discussions.

Received for publication November 6, 2003, and accepted in revised form August 3, 2004.

Address correspondence to: Jeffrey A. Bluestone, University of California at San Francisco, UCSF Diabetes Center, San Francisco, California 94143, USA. Phone: (415) 514-1683; Fax: (415) 5645813; E-mail: jbluest@diabetes.ucsf.edu.

Benoît L. Salomon's present address is: CNRS/UPMC UMR 7087, Hôpital Pitié-Salpêtrière, Paris, France. mice by CD154-independent antigen presentation to CD8(+) T cells. J. Exp. Med. 191:225-238.

9. Amrani, A., et al. 2002. CD154-dependent priming of diabetogenic CD4(+) T cells dissociated from activation of antigen-presenting cells. Immunity. 16:719-732.

10. Grewal, I.S., et al. 1996. Requirement for CD40 ligand in costimulation induction, $\mathrm{T}$ cell activation, and experimental allergic encephalomyelitis. Science. 273:1864-1867.

11. Yang, Y., and Wilson, J.M. 1996. CD40 ligand-dependent $\mathrm{T}$ cell activation: requirement of B7-CD28 signaling through CD40. Science. 273:1862-1864.

12. Sakaguchi, S. 2000. Regulatory T cells: key controllers of immunologic self-tolerance. Cell. 101:455-458.

13. Shevach, E.M. 2002. CD4+ CD25+ suppressor T cells: more questions than answers. Nat. Rev. Immunol. 2:389-400.

14. Salomon, B., et al. 2000. B7/CD28 costimulation is essential for the homeostasis of the CD4+CD25+ immunoregulatory $\mathrm{T}$ cells that control autoimmune diabetes. Immunity. 12:431-440.

15. Wu, A.J., Hua, H., Munson, S.H., and McDevitt, H.O. 2002. Tumor necrosis factor-alpha regulation of CD4+CD25+ T cell levels in NOD mice. Proc. Natl. Acad. Sci. U. S. A. 99:12287-12292.

16. Belghith, M., et al. 2003. TGF-beta-dependent mechanisms mediate restoration of self-tolerance induced by antibodies to CD3 in overt autoimmune diabetes. Nat. Med. 9:1202-1208.

17. Tang, Q., et al. 2003. CD28 Controls Peripheral Homeostasis of CD4(+)CD25(+) Regulatory T Cells. J. Immunol. 171:3348-3352.

18. Lenschow, D.J., et al. 1996. CD28/B7 regulation of Th1 and Th2 subsets in the development of autoimmune diabetes. Immunity. 5:285-293.

19. Katz, J.D., Wang, B., Haskins, K., Benoist, C., and Mathis, D. 1993. Following a diabetogenic $\mathrm{T}$ cell from genesis through pathogenesis. Cell. 74:1089-1100.

20. Lieberman, S.M., et al. 2003. Identification of the beta cell antigen targeted by a prevalent population of pathogenic CD8+ T cells in autoimmune diabetes. Proc. Natl. Acad. Sci. U. S. A. 100:8626-8628.

21. Lepault, F., and Gagnerault, M.C. 2000. Characterization of peripheral regulatory $\mathrm{CD} 4+\mathrm{T}$ cells that prevent diabetes onset in nonobese diabetic mice. J. Immunol. 164:240-247.

22. Szanya, V., Ermann, J., Taylor, C., Holness, C., and Fathman, C.G. 2002. The subpopulation of CD4+CD25+ splenocytes that delays adoptive transfer of diabetes expresses L-selectin and high levels of CCR7. J. Immunol. 169:2461-2465.

23. Karandikar, N.J., Vanderlugt, C.L., Walunas, T.L., Miller, S.D., and Bluestone, J.A. 1996. CTLA-4: a negative regulator of autoimmune disease. J. Exp. Med. 184:783-788. 
24. Walunas, T.L., and Bluestone, J.A. 1998. CTLA-4 regulates tolerance induction and $\mathrm{T}$ cell differentiation in vivo. J. Immunol. 160:3855-3860.

25. Punt, J.A., Havran, W., Abe, R., Sarin, A., and Singer, A. 1997. T cell receptor (TCR)-induced death of immature CD4+CD8+ thymocytes by two distinct mechanisms differing in their requirement for CD28 costimulation: implications for negative selection in the thymus. J. Exp. Med. 186:1911-1922.

26. Kishimoto, H., and Sprent, J. 1999. Several different cell surface molecules control negative selection of medullary thymocytes. J. Exp. Med. 190:65-73.

27. Lucas, B., and Germain, R.N. 2000. Opening a window on thymic positive selection: developmental changes in the influence of cosignaling by integrins and CD28 on selection events induced by TCR engagement. J. Immunol. 165:1889-1895.

28. Buhlmann, J.E., Elkin, S.K., and Sharpe, A.H. 2003. A role for the B7-1/B7-2:CD28/CTLA-4 pathway during negative selection. J. Immunol. 170:5421-5428.

29. Kundig, T.M., et al. 1996. Duration of TCR stimulation determines costimulatory requirement of $\mathrm{T}$ cells. Immunity. 5:41-52.

30. Lohr, J., Knoechel, B., Jiang, S., Sharpe, A.H., and Abbas, A.K. 2003. The inhibitory function of B7 costimulators in $\mathrm{T}$ cell responses to foreign and self-antigens. Nat. Immunol. 4:664-669.

31. Chambers, C.A., Kuhns, M.S., Egen, J.G., and Allison, J.P. 2001. CTLA-4-mediated inhibition in regulation of $\mathrm{T}$ cell responses: mechanisms and manipulation in tumor immunotherapy. Annu.
Rev. Immunol. 19:565-594.

32. Luhder, F., Chambers, C., Allison, J.P., Benoist, C., and Mathis, D. 2000. Pinpointing when T cell costimulatory receptor CTLA-4 must be engaged to dampen diabetogenic T cells. Proc. Natl. Acad. Sci. U. S. A. 97:12204-12209.

33. Mandelbrot, D.A., et al. 2001. B7-dependent T-cell costimulation in mice lacking CD28 and CTLA4. J. Clin. Invest. 107:881-887.

34. Apostolou, I., Sarukhan, A., Klein, L., and Von Boehmer, H. 2002. Origin of regulatory T cells with known specificity for antigen. Nat. Immunol. 3:756-763.

35. McHugh, R.S., and Shevach, E.M. 2002. Depletion of CD4+CD25+ regulatory $\mathrm{T}$ cells is necessary, but not sufficient, for induction of organ-specific autoimmune disease. J. Immunol. 168:5979-5983.

36. Klein, L., Khazaie, K., and von Boehmer, H. 2003. In vivo dynamics of antigen-specific regulatory $\mathrm{T}$ cells not predicted from behavior in vitro. Proc. Natl. Acad. Sci. U. S. A. 100:8886-8891.

37. Takahashi, T., et al. 1998. Immunologic self-tolerance maintained by CD25+CD4+ naturally anergic and suppressive $\mathrm{T}$ cells: induction of autoimmune disease by breaking their anergic/suppressive state. Int. Immunol. 10:1969-1980.

38. Thornton, A.M., and Shevach, E.M. 1998. CD4+CD25+ immunoregulatory $\mathrm{T}$ cells suppress polyclonal $\mathrm{T}$ cell activation in vitro by inhibiting interleukin 2 production. J. Exp. Med. 188:287-296.

39. Oldenhove, G., et al. 2003. CD4+ CD25+ Regula- tory $\mathrm{T}$ cells control T helper cell type 1 responses to foreign antigens induced by mature dendritic cells in vivo. J. Exp. Med. 198:259-266.

40. Herman, A.E., Freeman, G.J., Mathis, D., and Benoist, C. 2004. CD4+CD25+ T regulatory cells dependent on ICOS promote regulation of effector cells in the prediabetic lesion. J. Exp. Med. 199:1479-1489.

41. Kumanogoh, A., et al. 2001. Increased T cell autoreactivity in the absence of CD40-CD40 ligand interactions: a role of CD40 in regulatory T cell development. J. Immunol. 166:353-360.

42. Serra, P., et al. 2003. CD40 ligation releases immature dendritic cells from the control of regulatory CD4+CD25+ T cells. Immunity. 19:877-889.

43. Hong, S., et al. 2001. The natural killer T-cell ligand alpha-galactosylceramide prevents autoimmune diabetes in non-obese diabetic mice. Nat. Med. 7:1052-1056

44. Sharif, S., et al. 2001. Activation of natural killer $\mathrm{T}$ cells by alpha-galactosylceramide treatment prevents the onset and recurrence of autoimmune Type 1 diabetes. Nat. Med. 7:1057-1062.

45. Gonzalez, A., Andre-Schmutz, I., Carnaud, C., Mathis, D., and Benoist, C. 2001. Damage control, rather than unresponsiveness, effected by protective DX5+ T cells in autoimmune diabetes. Nat. Immunol. 2:1117-1125.

46. Jordan, M.S., et al. 2001. Thymic selection of $\mathrm{CD} 4+\mathrm{CD} 25+$ regulatory $\mathrm{T}$ cells induced by an agonist self-peptide. Nat. Immunol. 2:301-306. 